\title{
Generalized Analytic Fourier-Feynman Transform of Functionals in a Banach Algebra $\mathscr{F}_{A_{1}, A_{2}}^{a, b}$
}

\author{
Jae Gil Choi, ${ }^{1}$ David Skoug, ${ }^{2}$ and Seung Jun Chang ${ }^{1}$ \\ ${ }^{1}$ Department of Mathematics, Dankook University, Cheonan 330-714, Republic of Korea \\ ${ }^{2}$ Department of Mathematics, University of Nebraska-Lincoln, Lincoln, NE 68588-0130, USA \\ Correspondence should be addressed to Seung Jun Chang; sejchang@dankook.ac.kr
}

Received 18 July 2013; Accepted 26 September 2013

Academic Editor: Kari Ylinen

Copyright (C) 2013 Jae Gil Choi et al. This is an open access article distributed under the Creative Commons Attribution License, which permits unrestricted use, distribution, and reproduction in any medium, provided the original work is properly cited.

We introduce the Fresnel type class $\mathscr{F}_{A_{1}, A_{2}}^{a, b}$. We also establish the existence of the generalized analytic Fourier-Feynman transform for functionals in the Banach algebra $\mathscr{F}_{A_{1}, A_{2}}^{a, b}$.

\section{Introduction}

Let $H$ be a separable Hilbert space and let $\mathscr{M}(H)$ be the space of all complex-valued Borel measures on $H$. The Fourier transform of $\sigma$ in $\mathscr{M}(H)$ is defined by

$$
f(\sigma)\left(h^{\prime}\right) \equiv \widehat{\sigma}\left(h^{\prime}\right)=\int_{H} \exp \left\{i\left\langle h, h^{\prime}\right\rangle\right\} d \sigma(h), \quad h^{\prime} \in H .
$$

The set of all functions of the form (1) is denoted by $\mathscr{F}(H)$ and is called the Fresnel class of $H$. Let $(H, B, v)$ be an abstract Wiener space. It is known $[1,2]$ that each functional of the form (1) can be extended to $B$ uniquely by

$$
\widehat{\sigma}(x)=\int_{H} \exp \left\{i(h, x)^{\sim}\right\} d \sigma(h), \quad x \in B,
$$

where $(\cdot, \cdot)^{\sim}$ is a stochastic inner product between $H$ and $B$. The Fresnel class $\mathscr{F}(B)$ of $B$ is the space of (equivalence classes of) all functionals of the form (2). There has been a tremendous amount of papers and books in the literature on the Fresnel integral theory and Fresnel classes $\mathscr{F}(B)$ and $\mathscr{F}(H)$ on abstract Wiener and Hilbert spaces. For an elementary introduction see [3, Chapter 20].

Furthermore, in [1], Kallianpur and Bromley introduced a larger class $\mathscr{F}_{A_{1}, A_{2}}$ than the Fresnel class $\mathscr{F}(B)$ and showed the existence of the analytic Feynman integral of functionals in $\mathscr{F}_{A_{1}, A_{2}}$ for a successful treatment of certain physical problems by means of a Feynman integral. The Fresnel class $\mathscr{F}_{A_{1}, A_{2}}$ of $B^{2}$ is the space of (equivalence classes of) all functionals on $B^{2}$ of the following form:

$$
F\left(x_{1}, x_{2}\right)=\int_{H} \exp \left\{\sum_{j=1}^{2} i\left(A_{j}^{1 / 2} h, x_{j}\right)^{\sim}\right\} d \sigma(h),
$$

where $A_{1}$ and $A_{2}$ are bounded, nonnegative, and self-adjoint operators on $H$ and $\sigma \in \mathscr{M}(H)$.

In this paper we study the functionals $F$ of the form (3) with $\left(x_{1}, x_{2}\right)$ in a very general function space $C_{a, b}^{2}[0, T] \equiv$ $C_{a, b}[0, T] \times C_{a, b}[0, T]$. The function space $C_{a, b}[0, T]$, induced by generalized Brownian motion process, was introduced by Yeh $[4,5]$ and was used extensively in [6-13]. In this paper, we also construct a concrete theory of the generalized analytic Fourier-Feynman transform (GFFT) of functionals in a generalized Fresnel type class defined on $C_{a, b}^{2}[0, T]$. Other work involving GFFT theories on $C_{a, b}[0, T]$ include $[6,7,9$, $12,13]$.

The Wiener process used in $[1,2,14-17]$ is stationary in time and is free of drift while the stochastic process used in this paper, as well as in $[4,6-13,18]$, is nonstationary in time and is subject to a drift $a(t)$.

It turns out, as noted in Remark 7 below, that including a drift term $a(t)$ makes establishing the existence of the GFFT 
of functionals on $C_{a, b}^{2}[0, T]$ very difficult. However, when $a(t) \equiv 0$ and $b(t)=t$ on $[0, T]$, the general function space $C_{a, b}[0, T]$ reduces to the Wiener space $C_{0}[0, T]$.

\section{Definitions and Preliminaries}

Let $a(t)$ be an absolutely continuous real-valued function on $[0, T]$ with $a(0)=0, a^{\prime}(t) \in L^{2}[0, T]$, and let $b(t)$ be a strictly increasing, continuously differentiable real-valued function with $b(0)=0$ and $b^{\prime}(t)>0$ for each $t \in[0, T]$. The generalized Brownian motion process $Y$ determined by $a(t)$ and $b(t)$ is a Gaussian process with mean function $a(t)$ and covariance function $r(s, t)=\min \{b(s), b(t)\}$. For more details, see $[6,10,12]$. By Theorem 14.2 in [5], the probability measure $\mu$ induced by $Y$, taking a separable version, is supported by $C_{a, b}[0, T]$ (which is equivalent to the Banach space of continuous functions $x$ on $[0, T]$ with $x(0)=0$ under the sup norm). Hence, $\left(C_{a, b}[0, T], \mathscr{B}\left(C_{a, b}[0, T]\right), \mu\right)$ is the function space induced by $Y$ where $\mathscr{B}\left(C_{a, b}[0, T]\right)$ is the Borel $\sigma$-algebra of $C_{a, b}[0, T]$. We then complete this function space to obtain $\left(C_{a, b}[0, T], \mathscr{W}\left(C_{a, b}[0, T]\right), \mu\right)$, where $\mathscr{W}\left(C_{a, b}[0, T]\right)$ is the set of all Wiener measurable subsets of $C_{a, b}[0, T]$.

A subset $B$ of $C_{a, b}[0, T]$ is said to be scale-invariant measurable provided $\rho B$ is $\mathscr{W}\left(C_{a, b}[0, T]\right)$-measurable for all $\rho>0$, and a scale-invariant measurable set $N$ is said to be a scale-invariant null set provided $\mu(\rho N)=0$ for all $\rho>0$. A property that holds except on a scale-invariant null set is said to hold scale-invariant almost everywhere (s-a.e.). A functional $F$ is said to be scale-invariant measurable provided $F$ is defined on a scale-invariant measurable set and $F(\rho \cdot)$ is $\mathscr{W}\left(C_{a, b}[0, T]\right)$-measurable for every $\rho>0$. If two functionals $F$ and $G$ defined on $C_{a, b}[0, T]$ are equal s-a.e., we write $F \approx G$.

Let $L_{a, b}^{1,2}[0, T]$ be the space of Lebesgue measurable functions on $[0, T]$ given by

$$
\begin{aligned}
L_{a, b}^{1,2}[0, T]=\{v & \int_{0}^{T}|v(s)|^{2} d b(s)<\infty, \\
& \left.\int_{0}^{T}|v(s)| d|a|(s)<\infty\right\},
\end{aligned}
$$

where $|a|(\cdot)$ is the total variation function of $a(\cdot)$. Then $L_{a, b}^{1,2}[0, T]$ is a separable Hilbert space with inner product defined by

$$
\begin{aligned}
(u, v)_{L_{a, b}^{1,2}}= & \int_{0}^{T} u(t) v(t) d b(t) \\
& +\left(\int_{0}^{T} u(t) d a(t)\right)\left(\int_{0}^{T} v(t) d a(t)\right) .
\end{aligned}
$$

In particular, note that $\|u\|_{L_{a, b}^{1,2}} \equiv\left[(u, u)_{L_{a, b}^{1,2}}\right]^{1 / 2}=0$ if and only if $u(t)=0$ a.e. on $[0, T]$.

Let $\left\{\phi_{j}\right\}_{j=1}^{\infty}$ be a complete orthonormal set in $L_{a, b}^{1,2}[0, T]$, each of whose elements is of bounded variation on $[0, T]$ such that

$$
\int_{0}^{T} \phi_{j}(t) \phi_{k}(t) d b(t)= \begin{cases}0, & j \neq k \\ 1, & j=k\end{cases}
$$

Then for each $v \in L_{a, b}^{1,2}[0, T]$, the Paley-Wiener-Zygmund (PWZ) stochastic integral $\langle v, x\rangle$ is defined by the following formula:

$$
\langle v, x\rangle=\lim _{n \rightarrow \infty} \int_{0}^{T} \sum_{j=1}^{n}\left(v, \phi_{j}\right)_{a, b} \phi_{j}(t) d x(t)
$$

for all $x \in C_{a, b}[0, T]$ for which the limit exists; one can show that for each $v \in L_{a, b}^{1,2}[0, T]$, the PWZ stochastic integral $\langle v, x\rangle$ exists for $\mu$-a.e. $x \in C_{a, b}[0, T]$, and if $v$ is of bounded variation on $[0, T]$, then the PWZ stochastic integral $\langle v, x\rangle$ equals the Riemann-Stieltjes integral $\int_{0}^{T} v(t) d x(t)$ for s-a.e. $x \in C_{a, b}[0, T]$.

Remark 1. (1) For each $v \in L_{a, b}^{1,2}[0, T]$, the PWZ stochastic integral $\langle v, x\rangle$ is a Gaussian random variable on $C_{a, b}[0, T]$ with mean $\int_{0}^{T} v(s) d a(s)$ and variance $\int_{0}^{T} v^{2}(s) d b(s)$.

(2) For all $u, v \in L_{a, b}^{1,2}[0, T]$,

$$
\begin{aligned}
& \int_{C_{a, b}[0, T]}\langle u, x\rangle\langle v, x\rangle d \mu(x) \\
& =\int_{0}^{T} u(s) v(s) d b(s) \\
& \quad+\left(\int_{0}^{T} u(s) d a(s)\right)\left(\int_{0}^{T} v(s) d a(s)\right) .
\end{aligned}
$$

Hence, we see that for all $u, v \in L_{a, b}^{1,2}[0, T], \int_{0}^{T} u(s) v(s) d b(s)=$ 0 if and only if $\langle u, x\rangle$ and $\langle v, x\rangle$ are independent random variables.

The following Cameron-Martin subspace of $C_{a, b}[0, T]$ plays an important role throughout this paper.

Let

$$
\begin{gathered}
C_{a, b}^{\prime}[0, T]=\left\{w \in C_{a, b}[0, T]: w(t)=\int_{0}^{t} z(s) d b(s)\right. \\
\text { for some } \left.z \in L_{a, b}^{1,2}[0, T]\right\} .
\end{gathered}
$$

For $w \in C_{a, b}^{\prime}[0, T]$, let $D: C_{a, b}^{\prime}[0, T] \rightarrow L_{a, b}^{1,2}[0, T]$ be defined by the following formula:

$$
D w(t)=\frac{d \lambda_{w}}{d \lambda_{b}}(t)
$$

where $d \lambda_{w} / d \lambda_{b}$ denotes the Radon-Nikodym derivative of the signed measure $\lambda_{w}$ induced by $w$, with respect to the Borel-Stieltjes measure $\lambda_{b}$ induced by $b$. Then $C_{a, b}^{\prime} \equiv$ $C_{a, b}^{\prime}[0, T]$ with inner product

$$
\left(w_{1}, w_{2}\right)_{C_{a, b}^{\prime}}=\int_{C_{a, b}[0, T]}\left\langle D w_{1}, x\right\rangle\left\langle D w_{2}, x\right\rangle d \mu(x)
$$

is a separable Hilbert space. 
Using (8), we observe that the linear operator given by (10) is an isometry. In fact, the inverse operator $D^{-1}$ : $L_{a, b}^{1,2}[0, T] \rightarrow C_{a, b}^{\prime}[0, T]$ is given by

$$
\left(D^{-1} z\right)(t)=\int_{0}^{t} z(s) d b(s) .
$$

Moreover, the triple $\left(C_{a, b}^{\prime}[0, T], C_{a, b}[0, T], \mu\right)$ becomes an abstract Wiener space.

Throughout this paper, for $w \in C_{a, b}^{\prime}[0, T]$, we will use the notation $(w, x)^{\sim}$ instead of $\langle D w, x\rangle$. We also use the following notations for $w_{1}, w_{2}, w \in C_{a, b}^{\prime}[0, T]$ :

$$
\begin{gathered}
\left(w_{1}, w_{2}\right)_{b}=\int_{0}^{T} D w_{1}(t) D w_{2}(t) d b(t), \\
\|w\|_{b}=\sqrt{(w, w)_{b}} .
\end{gathered}
$$

Then $C_{a, b}^{\prime}[0, T]$ with the inner product given by (13) is also a separable Hilbert space. It is easy to see that the two norms $\|\cdot\|_{C_{a b}^{\prime}}$ and $\|\cdot\|_{b}$ are equivalent. Furthermore, we have the following assertions.

(i) $a(\cdot)$ is an element of $C_{a, b}^{\prime}[0, T]$.

(ii) For each $w \in C_{a, b}^{\prime}[0, T]$, the random variable $x \mapsto$ $(w, x)^{\sim}$ is Gaussian with mean $(w, a)_{b}$ and variance $\|w\|_{b}^{2}$.

(iii) $(w, \alpha x)^{\sim}=(\alpha w, x)^{\sim}=\alpha(w, x)^{\sim}$ for any real number $\alpha, w \in C_{a, b}^{\prime}[0, T]$ and $x \in C_{a, b}[0, T]$.

(iv) Let $\left\{w_{1}, \ldots, w_{n}\right\}$ be a subset of $C_{a, b}^{\prime}[0, T]$ such that $\int_{0}^{T} D w_{i}(t) D w_{j}(t) d b(t)=\delta_{i j}$, where $\delta_{i j}$ is the Kronecker delta. Then the random variables $\left(w_{i}, x\right)^{\sim}$ 's are independent.

In this paper, we adopt as much as possible the definitions and notations used in $[7,9,12,13]$ for the definitions of the generalized analytic Feynman integral and the GFFT of functionals on $C_{a, b}[0, T]$.

The following integration formula is used several times in this paper:

$$
\int_{\mathbb{R}} \exp \left\{-\alpha u^{2}+\beta u\right\} d u=\sqrt{\frac{\pi}{\alpha}} \exp \left\{\frac{\beta^{2}}{4 \alpha}\right\}
$$

for complex numbers $\alpha$ and $\beta$ with $\operatorname{Re}(\alpha)>0$.

\section{The GFFT of Functionals in a Banach Algebra $\mathscr{F}_{A_{1}, A_{2}}^{a, b}$}

Let $\mathscr{M}\left(C_{a, b}^{\prime}[0, T]\right)$ be the space of complex-valued, countably additive (and hence finite) Borel measures on $C_{a, b}^{\prime}[0, T]$. $\mathscr{M}\left(C_{a, b}^{\prime}[0, T]\right)$ is a Banach algebra under the total variation norm and with convolution as multiplication.

We define the Fresnel type class $\mathscr{F}\left(C_{a, b}[0, T]\right)$ of functionals on $C_{a, b}[0, T]$ as the space of all stochastic Fourier transforms of elements of $\mathscr{M}\left(C_{a, b}^{\prime}[0, T]\right)$; that is,
$F \in \mathscr{F}\left(C_{a, b}[0, T]\right)$ if and only if there exists a measure $f$ in $\mathscr{M}\left(C_{a, b}^{\prime}[0, T]\right)$ such that

$$
F(x)=\int_{C_{a, b}^{\prime}[0, T]} \exp \left\{i(w, x)^{\sim}\right\} d f(w)
$$

for s-a.e. $x \in C_{a, b}[0, T]$. More precisely, since we will identify functionals which coincide s-a.e. on $C_{a, b}[0, T], \mathscr{F}\left(C_{a, b}[0, T]\right)$ can be regarded as the space of all $s$-equivalence classes of functionals of the form (15).

The Fresnel type class $\mathscr{F}\left(C_{a, b}[0, T]\right)$ is a Banach algebra with norm

$$
\|F\|=\|f\|=\int_{C_{a, b}^{\prime}[0, T]} d|f|(w) .
$$

In fact, the correspondence $f \mapsto F$ is injective, carries convolution into pointwise multiplication and is a Banach algebra isomorphism where $f$ and $F$ are related by (15).

Remark 2. The Banach algebra $\mathscr{F}\left(C_{a, b}[0, T]\right)$ contains several interesting functions which arise naturally in quantum mechanics. Let $\mathscr{M}(\mathbb{R})$ be the class of $\mathbb{C}$-valued countably additive measures on $\mathscr{B}(\mathbb{R})$, the Borel class of $\mathbb{R}$. For $\nu \in$ $\mathscr{M}(\mathbb{R})$, the Fourier transform $\hat{v}$ of $\nu$ is a complex-valued function defined on $\mathbb{R}$ by the following formula:

$$
\widehat{\nu}(u)=\int_{\mathbb{R}} \exp \{i u v\} d \nu(v) .
$$

Let $\mathscr{G}$ be the set of all complex-valued functions on $[0, T] \times \mathbb{R}$ of the form $\theta(s, u)=\widehat{\sigma}_{s}(u)$, where $\left\{\sigma_{s}: 0 \leq s \leq T\right\}$ is a family from $\mathscr{M}(\mathbb{R})$ satisfying the following two conditions:

(i) for every $E \in \mathscr{B}(\mathbb{R}), \sigma_{s}(E)$ is Borel measurable in $s$,

(ii) $\int_{0}^{T}\left\|\sigma_{s}\right\| d b(s)<+\infty$.

Let $\theta \in \mathscr{G}$ and let $H$ be given by

$$
H(x)=\exp \left\{\int_{0}^{T} \theta(t, x(t)) d t\right\}
$$

for s-a.e. $x \in C_{a, b}[0, T]$. Then, using the methods similar to those used in [18], we can show that the function $\theta(t, u)$ is Borel-measurable and that $\theta(t, x(t)), \int_{0}^{T} \theta(t, x(t)) d t$, and $H(x)$ are elements of $\mathscr{F}\left(C_{a, b}[0, T]\right)$. These facts are relevant to quantum mechanics where exponential functions play a prominent role.

Let $A$ be a nonnegative self-adjoint operator on $C_{a, b}^{\prime}[0, T]$ and $f$ any complex measure on $C_{a, b}^{\prime}[0, T]$. Then the functional

$$
F(x)=\int_{C_{a, b}^{\prime}[0, T]} \exp \left\{i\left(A^{1 / 2} w, x\right)^{\sim}\right\} d f(w)
$$

belongs to $\mathscr{F}\left(C_{a, b}[0, T]\right)$ because it can be rewritten as $\int_{C_{a, b}^{\prime}[0, T]} \exp \left\{i(w, x)^{\sim}\right\} d f_{A}(w)$ for $f_{A}=f \circ\left(A^{1 / 2}\right)^{-1}$. Let $A$ be self-adjoint but not nonnegative. Then $A$ has the form

$$
A=A^{+}-A^{-},
$$


where both $A^{+}$and $A^{-}$are bounded, nonnegative, and selfadjoint operators.

In this section we will extend the ideas of [1] to obtain expressions of the generalized analytic Feynman integral and the GFFT of functionals of the form (19) when $A$ is no longer required to be nonnegative. To do this, we will introduce definitions and notations analogous to those in $[7,12,13]$.

Let $\mathscr{W}\left(C_{a, b}^{2}[0, T]\right)$ denote the class of all Wiener measurable subsets of the product function space $C_{a, b}^{2}[0, T]$. A subset $B$ of $C_{a, b}^{2}[0, T]$ is said to be scale-invariant measurable provided $\left\{\left(\rho_{1} x_{1}, \rho_{2} x_{2}\right):\left(x_{1}, x_{2}\right) \in B\right\}$ is $\mathscr{W}\left(C_{a, b}^{2}[0, T]\right)$ measurable for every $\rho_{1}>0$ and $\rho_{2}>0$, and a scaleinvariant measurable subset $N$ of $C_{a, b}^{2}[0, T]$ is said to be scaleinvariant null provided $(\mu \times \mu)\left(\left\{\left(\rho_{1} x_{1}, \rho_{2} x_{2}\right):\left(x_{1}, x_{2}\right) \in\right.\right.$ $N\})=0$ for every $\rho_{1}>0$ and $\rho_{2}>0$. A property that holds except on a scale-invariant null set is said to hold sa.e. on $C_{a, b}^{2}[0, T]$. A functional $F$ on $C_{a, b}^{2}[0, T]$ is said to be scale-invariant measurable provided $F$ is defined on a scaleinvariant measurable set and $F\left(\rho_{1} \cdot, \rho_{2} \cdot\right)$ is $\mathscr{W}\left(C_{a, b}^{2}[0, T]\right)$ measurable for every $\rho_{1}>0$ and $\rho_{2}>0$. If two functionals $F$ and $G$ defined on $C_{a, b}^{2}[0, T]$ are equal s-a.e., then we write $F \approx G$.

We denote the product function space integral of a $\mathscr{W}\left(C_{a, b}^{2}[0, T]\right)$-measurable functional $F$ by

$$
\begin{aligned}
E[F] & \equiv E_{\vec{x}}\left[F\left(x_{1}, x_{2}\right)\right] \\
& =\int_{C_{a, b}^{2}[0, T]} F\left(x_{1}, x_{2}\right) d(\mu \times \mu)\left(x_{1}, x_{2}\right)
\end{aligned}
$$

whenever the integral exists.

Throughout this paper, let $\mathbb{C}, \mathbb{C}_{+}$and $\widetilde{\mathbb{C}}_{+}$denote the set of complex numbers, complex numbers with positive real part, and nonzero complex numbers with nonnegative real part, respectively. Furthermore, for all $\lambda \in \widetilde{\mathbb{C}}_{+}, \lambda^{-1 / 2}$ (or $\lambda^{1 / 2}$ ) is always chosen to have positive real part. We also assume that every functional $F$ on $C_{a, b}^{2}[0, T]$ we consider is s-a.e. defined and is scale-invariant measurable.

Definition 3. Let $\mathbb{C}_{+}^{2} \equiv \mathbb{C}_{+} \times \mathbb{C}_{+}$and let $\widetilde{\mathbb{C}}_{+}^{2} \equiv \widetilde{\mathbb{C}}_{+} \times \widetilde{\mathbb{C}}_{+}$. Let $F: C_{a, b}^{2}[0, T] \rightarrow \mathbb{C}$ be such that for each $\lambda_{1}>0$ and $\lambda_{2}>0$, the function space integral

$$
\begin{aligned}
& J\left(\lambda_{1}, \lambda_{2}\right) \\
& \quad=\int_{C_{a, b}^{2}[0, T]} F\left(\lambda_{1}^{-1 / 2} x_{1}, \lambda_{2}^{-1 / 2} x_{2}\right) d(\mu \times \mu)\left(x_{1}, x_{2}\right)
\end{aligned}
$$

exists. If there exists a function $J^{*}\left(\lambda_{1}, \lambda_{2}\right)$ analytic in $\mathbb{C}_{+}^{2}$ such that $J^{*}\left(\lambda_{1}, \lambda_{2}\right)=J\left(\lambda_{1}, \lambda_{2}\right)$ for all $\lambda_{1}>0$ and $\lambda_{2}>0$, then $J^{*}\left(\lambda_{1}, \lambda_{2}\right)$ is defined to be the analytic function space integral of $F$ over $C_{a, b}^{2}[0, T]$ with parameter $\vec{\lambda}=\left(\lambda_{1}, \lambda_{2}\right)$, and for $\vec{\lambda} \epsilon$ $\mathbb{C}_{+}^{2}$ we write

$$
\begin{aligned}
E^{\mathrm{an}_{\vec{\lambda}}}[F] & \equiv E_{\vec{x}}^{\mathrm{an}_{\vec{\lambda}}}\left[F\left(x_{1}, x_{2}\right)\right] \\
& \equiv E_{x_{1}, x_{2}}^{\mathrm{an}_{\left(\lambda_{1}, \lambda_{2}\right)}}\left[F\left(x_{1}, x_{2}\right)\right]=J^{*}\left(\lambda_{1}, \lambda_{2}\right) .
\end{aligned}
$$

Let $q_{1}$ and $q_{2}$ be nonzero real numbers. Let $F$ be a functional such that $E^{\mathrm{an}} \vec{\lambda}[F]$ exists for all $\vec{\lambda} \in \mathbb{C}_{+}^{2}$. If the following limit exists, we call it the generalized analytic Feynman integral of $F$ with parameter $\vec{q}=\left(q_{1}, q_{2}\right)$, and we write

$$
\begin{aligned}
E^{\operatorname{anf} f_{\vec{q}}}[F] & \equiv E_{\vec{x}}^{\operatorname{anf}_{\vec{q}}}\left[F\left(x_{1}, x_{2}\right)\right] \\
& \equiv E_{x_{1}, x_{2}}^{\operatorname{anf}_{\left(q_{1}, q_{2}\right)}}\left[F\left(x_{1}, x_{2}\right)\right]=\lim _{\vec{\lambda} \rightarrow-i \vec{q}} E^{\operatorname{an}_{\vec{\lambda}}}[F],
\end{aligned}
$$

where $\vec{\lambda}=\left(\lambda_{1}, \lambda_{2}\right) \rightarrow-i \vec{q}=\left(-i q_{1},-i q_{2}\right)$ through values in $\mathbb{C}_{+}^{2}$.

Definition 4. Let $q_{1}$ and $q_{2}$ be nonzero real numbers. For $\vec{\lambda}=$ $\left(\lambda_{1}, \lambda_{2}\right) \in \mathbb{C}_{+}^{2}$ and $\left(y_{1}, y_{2}\right) \in C_{a, b}^{2}[0, T]$, let

$$
\begin{aligned}
T_{\vec{\lambda}}(F)\left(y_{1}, y_{2}\right) & \equiv T_{\left(\lambda_{1}, \lambda_{2}\right)}(F)\left(y_{1}, y_{2}\right) \\
& =E_{\vec{x}}^{\mathrm{an}_{\vec{\lambda}}}\left[F\left(y_{1}+x_{1}, y_{2}+x_{2}\right)\right] .
\end{aligned}
$$

For $p \in(1,2]$, we define the $L_{p}$ analytic GFFT, $T_{\vec{q}}^{(p)}(F)$ of $F$, by the formula $\left(\vec{\lambda} \in \mathbb{C}_{+}^{2}\right)$

$$
\begin{aligned}
T_{\vec{q}}^{(p)}(F)\left(y_{1}, y_{2}\right) & \equiv T_{\left(q_{1}, q_{2}\right)}^{(p)}(F)\left(y_{1}, y_{2}\right) \\
& =\lim _{\vec{\lambda} \rightarrow-i \vec{q}} T_{\vec{\lambda}}(F)\left(y_{1}, y_{2}\right)
\end{aligned}
$$

if it exists; that is, for each $\rho_{1}>0$ and $\rho_{2}>0$,

$$
\begin{aligned}
\lim _{\vec{\lambda} \rightarrow-i \vec{q}} \int_{C_{a, b}^{2}[0, T]} \mid & T_{\vec{\lambda}}(F)\left(\rho_{1} y_{1}, \rho_{2} y_{2}\right) \\
& -\left.T_{\vec{q}}^{(p)}(F)\left(\rho_{1} y_{1}, \rho_{2} y_{2}\right)\right|^{p^{\prime}} d(\mu \times \mu)\left(y_{1}, y_{2}\right)
\end{aligned}
$$$$
=0 \text {, }
$$

where $1 / p+1 / p^{\prime}=1$. We define the $L_{1}$ analytic GFFT, $T_{\vec{q}}^{(1)}(F)$ of $F$, by the formula $\left(\vec{\lambda} \in \mathbb{C}_{+}^{2}\right)$

$$
T_{\vec{q}}^{(1)}(F)\left(y_{1}, y_{2}\right)=\lim _{\vec{\lambda} \rightarrow-i \vec{q}} T_{\vec{\lambda}}(F)\left(y_{1}, y_{2}\right)
$$

if it exists.

We note that for $1 \leq p \leq 2, T_{\vec{q}}^{(p)}(F)$ is defined only s-a.e. We also note that if $T_{\vec{q}}^{(p)}(F)$ exists and if $F \approx G$, then $T_{\vec{q}}^{(p)}(G)$ exists and $T_{\vec{q}}^{(p)}(G) \approx T_{\vec{q}}^{(p)}(F)$. Moreover, from Definition 4, we see that for $q_{1}, q_{2} \in \mathbb{R}-\{0\}$

$$
E_{\vec{x}}^{\operatorname{anf}_{\vec{q}}}\left[F\left(x_{1}, x_{2}\right)\right]=T_{\vec{q}}^{(1)}(F)(0,0) .
$$

Next we give the definition of the generalized Fresnel type class $\mathscr{F}_{A_{1}, A_{2}}^{a, b}$. 
Definition 5. Let $A_{1}$ and $A_{2}$ be bounded, nonnegative, and self-adjoint operators on $C_{a, b}^{\prime}[0, T]$. The generalized Fresnel type class $\mathscr{F}_{A_{1}, A_{2}}^{a, b}$ of functionals on $C_{a, b}^{2}[0, T]$ is defined as the space of all functionals $F$ on $C_{a, b}^{2}[0, T]$ of the following form:

$$
F\left(x_{1}, x_{2}\right)=\int_{C_{a, b}^{\prime}[0, T]} \exp \left\{\sum_{j=1}^{2} i\left(A_{j}^{1 / 2} w, x_{j}\right)^{\sim}\right\} d f(w)
$$

for some $f \in \mathscr{M}\left(C_{a, b}^{\prime}[0, T]\right)$. More precisely, since we identify functionals which coincide s-a.e. on $C_{a, b}^{2}[0, T], \mathscr{F}_{A_{1}, A_{2}}^{a, b}$ can be regarded as the space of all $s$-equivalence classes of functionals of the form (30).

Remark 6. (1) In Definition 5, let $A_{1}$ be the identity operator on $C_{a, b}^{\prime}[0, T]$ and $A_{2} \equiv 0$. Then $\mathscr{F}_{A_{1}, A_{2}}^{a, b}$ is essentially the Fresnel type class $\mathscr{F}\left(C_{a, b}[0, T]\right)$, and for $p \in[1,2]$ and nonzero real numbers $q_{1}$ and $q_{2}$,

$$
T_{\left(q_{1}, q_{2}\right)}^{(p)}(F)\left(y_{1}, y_{2}\right)=T_{q_{1}}^{(p)}\left(F_{0}\right)\left(y_{1}\right)
$$

if it exists, where $F_{0}\left(x_{1}\right)=F\left(x_{1}, x_{2}\right)$ for all $\left(x_{1}, x_{2}\right) \in$ $C_{a, b}^{2}[0, T]$ and $T_{q_{1}}^{(p)}\left(F_{0}\right)$ means the $L_{p}$ analytic GFFT on $C_{a, b}[0, T]$; see $[6,12]$.

(2) The map $f \mapsto F$ defined by (30) sets up an algebra isomorphism between $\mathscr{M}\left(C_{a, b}^{\prime}[0, T]\right)$ and $\mathscr{F}_{A_{1}, A_{2}}^{a, b}$ if $\operatorname{Ran}\left(A_{1}+\right.$ $\left.A_{2}\right)$ is dense in $C_{a, b}^{\prime}[0, T]$, where Ran indicates the range of an operator. In this case $\mathscr{F}_{A_{1}, A_{2}}^{a, b}$ becomes a Banach algebra under the norm $\|F\|=\|f\|$. For more details see [1].

Remark 7. Let $F$ be given by (30). In evaluating $E_{\vec{x}}\left[F\left(\lambda_{1}^{-1 / 2} x_{1}, \lambda_{2}^{-1 / 2} x_{2}\right)\right]$ and $T_{\left(\lambda_{1}, \lambda_{2}\right)}(F)\left(y_{1}, y_{2}\right)=E_{\vec{x}}\left[F\left(y_{1}+\right.\right.$ $\left.\left.\lambda_{1}^{-1 / 2} x_{1}, y_{2}+\lambda_{2}^{-1 / 2} x_{2}\right)\right]$ for $\lambda_{1}>0$ and $\lambda_{2}>0$, the expression

$$
\begin{aligned}
& \psi(\vec{\lambda} ; \vec{A} ; w) \\
& \quad \equiv \psi\left(\lambda_{1}, \lambda_{2} ; A_{1}, A_{2} ; w\right) \\
& \quad=\exp \left\{\sum_{j=1}^{2}\left[-\frac{\left(A_{j} w, w\right)_{b}}{2 \lambda_{j}}+i \lambda_{j}^{-1 / 2}\left(A_{j}^{1 / 2} w, a\right)_{b}\right]\right\}
\end{aligned}
$$

occurs. Clearly, for $\lambda_{j}>0, j \in\{1,2\},|\psi(\vec{\lambda} ; \vec{A} ; w)| \leq 1$ for all $w \in C_{a, b}^{\prime}[0, T]$. But for $\vec{\lambda} \in \widetilde{\mathbb{C}}_{+}^{2},|\psi(\vec{\lambda} ; \vec{A} ; w)|$ is not necessarily bounded by 1 .

Note that for each $\lambda_{j} \in \widetilde{\mathbb{C}}_{+}$with $\lambda_{j}=\alpha_{j}+i \beta_{j}, j \in\{1,2\}$,

$$
\begin{aligned}
& \lambda_{j}^{1 / 2}=\sqrt{\frac{\sqrt{\alpha_{j}^{2}+\beta_{j}^{2}}+\alpha_{j}}{2}}+i \frac{\beta_{j}}{\left|\beta_{j}\right|} \sqrt{\frac{\sqrt{\alpha_{j}^{2}+\beta_{j}^{2}}-\alpha_{j}}{2}}, \\
& \lambda_{j}^{-1 / 2}=\sqrt{\frac{\sqrt{\alpha_{j}^{2}+\beta_{j}^{2}}+\alpha_{j}}{2\left(\alpha_{j}^{2}+\beta_{j}^{2}\right)}}-i \frac{\beta_{j}}{\left|\beta_{j}\right|} \sqrt{\frac{\sqrt{\alpha_{j}^{2}+\beta_{j}^{2}}-\alpha_{j}}{2\left(\alpha_{j}^{2}+\beta_{j}^{2}\right)}} .
\end{aligned}
$$

Hence, for $\lambda_{j} \in \widetilde{\mathbb{C}}_{+}$with $\lambda_{j}=\alpha_{j}+i \beta_{j}, j \in\{1,2\}$,

$$
\begin{aligned}
|\psi(\vec{\lambda} ; \vec{A} ; w)| & \exp \left\{\sum _ { j = 1 } ^ { 2 } \left[-\frac{\alpha_{j}}{2\left(\alpha_{j}^{2}+\beta_{j}^{2}\right)}\left(A_{j} w, w\right)_{b}\right.\right. \\
& +\frac{\beta_{j}}{\left|\beta_{j}\right|} \sqrt{\left.\left.\frac{\sqrt{\alpha_{j}^{2}+\beta_{j}^{2}}-\alpha_{j}}{2\left(\alpha_{j}^{2}+\beta_{j}^{2}\right)}\left(A_{j}^{1 / 2} w, a\right)_{b}\right]\right\} .}
\end{aligned}
$$

The right hand side of (34) is an unbounded function of $w$ for $w \in C_{a, b}^{\prime}[0, T]$. Thus $E^{\mathrm{an}_{\vec{\lambda}}}[F], E^{\operatorname{anf}_{\vec{q}}}[F], T_{\vec{\lambda}}(F)$, and $T_{\vec{q}}^{(p)}(F)$ might not exist. Thus throughout this paper we will need to put additional restrictions on the complex measure $f$ corresponding to $F$ in order to obtain our results for the GFFT and the generalized analytic Feynman integral of $F$.

In view of Remark 7, we clearly need to impose additional restrictions on the functionals $F$ in $\mathscr{F}_{A_{1}, A_{2}}^{a, b}$.

For a positive real number $q_{0}$, let

$$
\begin{gathered}
\Gamma_{q_{0}}=\left\{\vec{\lambda}=\left(\lambda_{1}, \lambda_{2}\right) \in \widetilde{\mathbb{C}}_{+}^{2} \mid \lambda_{j}=\alpha_{j}+i \beta_{j},\right. \\
\left|\operatorname{Im}\left(\lambda_{j}^{-1 / 2}\right)\right|=\sqrt{\frac{\sqrt{\alpha_{j}^{2}+\beta_{j}^{2}}-\alpha_{j}}{2\left(\alpha_{j}^{2}+\beta_{j}^{2}\right)}} \\
\left.<\frac{1}{\sqrt{2 q_{0}}}, j=1,2\right\}
\end{gathered}
$$

and let

$$
\begin{aligned}
k\left(q_{0} ; \vec{A} ; w\right) & \equiv k\left(q_{0} ; A_{1}, A_{2} ; w\right) \\
& =\exp \left\{\sum_{j=1}^{2}\left(2 q_{0}\right)^{-1 / 2}\left\|A_{j}^{1 / 2} w\right\|_{b}\|a\|_{b}\right\} .
\end{aligned}
$$

Then, for all $\vec{\lambda}=\left(\lambda_{1}, \lambda_{2}\right) \in \Gamma_{q_{0}}$

$$
\begin{aligned}
|\psi(\vec{\lambda} ; \vec{A} ; w)| & \leq \exp \left\{\sum_{j=1}^{2} \sqrt{\frac{\sqrt{\alpha_{j}^{2}+\beta_{j}^{2}}-\alpha_{j}}{2\left(\alpha_{j}^{2}+\beta_{j}^{2}\right)}}\left|\left(A_{j}^{1 / 2} w, a\right)_{b}\right|\right\} \\
& \leq \exp \left\{\sum_{j=1}^{2} \sqrt{\frac{\sqrt{\alpha_{j}^{2}+\beta_{j}^{2}}-\alpha_{j}}{2\left(\alpha_{j}^{2}+\beta_{j}^{2}\right)}}\left\|A_{j}^{1 / 2} w\right\|_{b}\|a\|_{b}\right\} \\
& <k\left(q_{0} ; \vec{A} ; w\right) .
\end{aligned}
$$


We note that for all real $q_{j}$ with $\left|q_{j}\right|>q_{0}, j \in\{1,2\}$,

$$
\left(-i q_{j}\right)^{-1 / 2}=\frac{1}{\sqrt{\left|2 q_{j}\right|}}+\operatorname{sign}\left(q_{j}\right) \frac{i}{\sqrt{\left|2 q_{j}\right|}}
$$

and $\left(-i q_{1},-i q_{2}\right) \in \Gamma_{q_{0}}$.

For the existence of the GFFT of $F$, we define a subclass $\mathscr{F}_{A_{1}, A_{2}}^{q_{0}}$ of $\mathscr{F}_{A_{1}, A_{2}}^{a, b}$ by $F \in \mathscr{F}_{A_{1}, A_{2}}^{q_{0}}$ if and only if

$$
\int_{C_{a, b}^{\prime}[0, T]} k\left(q_{0} ; \vec{A} ; w\right) d|f|(w)<+\infty,
$$

where $f$ and $F$ are related by (30) and $k$ is given by (36).

Remark 8. Note that in case $a(t) \equiv 0$ and $b(t)=t$ on $[0, T]$, the function space $C_{a, b}[0, T]$ reduces to the classical Wiener space $C_{0}[0, T]$ and $(w, a)_{b}=0$ for all $w \in C_{a, b}^{\prime}[0, T]=$ $C_{0}^{\prime}[0, T]$. Hence, for all $\vec{\lambda} \in \widetilde{\mathbb{C}}_{+}^{2},|\psi(\vec{\lambda} ; \vec{A} ; w)| \leq 1$ and for any positive real number $q_{0}, \mathscr{F}_{A_{1}, A_{2}}^{q_{0}}=\mathscr{F}_{A_{1}, A_{2}}$, the Kallianpur and Bromley's class introduced in Section 1.

Theorem 9. Let $q_{0}$ be a positive real number and let $F$ be an element of $\mathscr{F}_{A_{1}, A_{2}}^{q_{0}}$. Then for any nonzero real numbers $q_{1}$ and $q_{2}$ with $\left|q_{j}\right|>q_{0}, j \in\{1,2\}$, the $L_{1}$ analytic GFFT of $F, T_{\vec{q}}^{(1)}(F)$ exists and is given by the following formula:

$$
\begin{aligned}
T_{\vec{q}}^{(1)} & (F)\left(y_{1}, y_{2}\right) \\
\quad= & \int_{C_{a, b}^{\prime}[0, T]} \exp \left\{\sum_{j=1}^{2} i\left(A_{j}^{1 / 2} w, y_{j}\right)^{\sim}\right\} \psi(-i \vec{q} ; \vec{A} ; w) d f(w)
\end{aligned}
$$

for s-a.e. $\left(y_{1}, y_{2}\right) \in C_{a, b}^{2}[0, T]$, where $\psi$ is given by (32).

Proof. We first note that for $j \in\{1,2\}$, the PWZ stochastic integral $\left(A_{j}^{1 / 2} w, x\right)^{\sim}$ is a Gaussian random variable with mean $\left(A_{j}^{1 / 2} w, a\right)_{b}$ and variance $\left\|A_{j}^{1 / 2} w\right\|_{b}^{2}=\left(A_{j} w, w\right)_{b}$. Hence, using (30), the Fubini theorem, the change of variables theorem and (14), we have that for all $\lambda_{1}>0$ and $\lambda_{2}>0$,

$$
\begin{aligned}
& J\left(y_{1}, y_{2} ; \lambda_{1}, \lambda_{2}\right) \\
& \equiv E_{\vec{x}}\left[F\left(y_{1}+\lambda_{1}^{-1 / 2} x_{1}, y_{2}+\lambda_{2}^{-1 / 2} x_{2}\right)\right] \\
& =\int_{C_{a, b}^{\prime}[0, T]} \exp \left\{\sum_{j=1}^{2} i\left(A_{j}^{1 / 2} w, y_{j}\right)^{\sim}\right\} \\
& \quad \times\left(\prod_{j=1}^{2} E_{x_{j}}\left[\exp \left\{i \lambda_{j}^{-1 / 2}\left(A_{j}^{1 / 2} w, x_{j}\right)^{\sim}\right\}\right]\right) d f(w)
\end{aligned}
$$

$$
\begin{aligned}
&=\int_{C_{a, b}^{\prime}[0, T]} \exp \left\{\sum_{j=1}^{2} i\left(A_{j}^{1 / 2} w, y_{j}\right)^{\sim}\right\} \\
& \times {\left[\prod_{j=1}^{2}\left(2 \pi\left(A_{j} w, w\right)_{b}\right)^{-1 / 2}\right.} \\
& \times \int_{\mathbb{R}} \exp \left\{i \lambda_{j}^{-1 / 2} u_{j}\right. \\
&=\int_{C_{a, b}^{\prime}[0, T]} \exp \left\{\sum_{j=1}^{2} i\left(A_{j}^{1 / 2} w, y_{j}\right)^{\sim}\right\} \psi(\vec{\lambda} ; \vec{A} ; w) d f(w) .
\end{aligned}
$$

Let

$$
\begin{aligned}
& T_{\vec{\lambda}}(F)\left(y_{1}, y_{2}\right) \\
& =\int_{C_{a, b}^{\prime}[0, T]} \exp \left\{\sum_{j=1}^{2} i\left(A_{j}^{1 / 2} w, y_{j}\right)^{\sim}\right\} \psi(\vec{\lambda} ; \vec{A} ; w) d f(w)
\end{aligned}
$$

for each $\vec{\lambda} \in \mathbb{C}_{+}^{2}$. Clearly,

$$
T_{\vec{\lambda}}(F)\left(y_{1}, y_{2}\right)=J\left(y_{1}, y_{2} ; \lambda_{1}, \lambda_{2}\right)
$$

for all $\lambda_{1}>0$ and $\lambda_{2}>0$. Let $\Gamma_{q_{0}}$ be given by (35). Then for all $\vec{\lambda} \in \operatorname{Int}\left(\Gamma_{q_{0}}\right)$

$$
\left|T_{\vec{\lambda}}(F)\left(y_{1}, y_{2}\right)\right|<\int_{C_{a, b}^{\prime}[0, T]} k\left(q_{0} ; \vec{A} ; w\right) d|f|(w)<+\infty .
$$

Using this fact and the dominated convergence theorem, we see that $T_{\vec{\lambda}}(F)\left(y_{1}, y_{2}\right)$ is a continuous function of $\vec{\lambda}=$ $\left(\lambda_{1}, \lambda_{2}\right)$ on $\operatorname{Int}\left(\Gamma_{q_{0}}\right)$. For each $w \in C_{a, b}^{\prime}[0, T], \psi(\vec{\lambda} ; \vec{A} ; w)$ is an analytic function of $\vec{\lambda}$ throughout the $\operatorname{domain} \operatorname{Int}\left(\Gamma_{q_{0}}\right)$ so that $\int_{\Delta} \psi(\vec{\lambda} ; \vec{A} ; w) d \vec{\lambda}=0$ for every rectifiable simple closed curve $\Delta$ in $\operatorname{Int}\left(\Gamma_{q_{0}}\right)$. By (42), the Fubini theorem and the Morera theorem, we see that $T_{\vec{\lambda}}(F)\left(y_{1}, y_{2}\right)$ is an analytic function of $\vec{\lambda}$ throughout the domain $\operatorname{Int}\left(\Gamma_{q_{0}}\right)$. Finally, using (28) with the dominated convergence theorem, we obtain the desired result.

Theorem 10. Let $q_{0}$ and $F$ be as in Theorem 9. Then for all $p \in$ $(1,2]$ and all nonzero real numbers $q_{1}$ and $q_{2}$ with $\left|q_{j}\right|>q_{0}$, $j \in\{1,2\}$, the $L_{p}$ analytic GFFT of $F, T_{\vec{q}}^{(p)}(F)$ exists and is given by the right hand side of (40) for s-a.e. $\left(y_{1}, y_{2}\right) \in C_{a, b}^{2}[0, T]$. 
Proof. Let $\Gamma_{q_{0}}$ be given by (35). It was shown in the proof of Theorem 9 that $T_{\vec{\lambda}}(F)\left(y_{1}, y_{2}\right)$ is an analytic function of $\vec{\lambda}$ throughout the domain $\operatorname{Int}\left(\Gamma_{q_{0}}\right)$. In view of Definition 4 , it will suffice to show that for each $\rho_{1}>0$ and $\rho_{2}>0$,

$$
\begin{gathered}
\lim _{\vec{\lambda} \rightarrow-i \vec{q}} \int_{C_{a, b}^{2}[0, T]} \mid T_{\vec{\lambda}}(F)\left(\rho_{1} y_{1}, \rho_{2} y_{2}\right) \\
-\left.T_{\vec{q}}^{(p)}(F)\left(\rho_{1} y_{1}, \rho_{2} y_{2}\right)\right|^{p^{\prime}} \\
\times d(\mu \times \mu)\left(y_{1}, y_{2}\right)=0 .
\end{gathered}
$$

Fixing $p \in(1,2]$ and using the inequalities (37) and (39), we obtain that for all $\rho_{j}>0, j \in\{1,2\}$ and all $\vec{\lambda} \in \Gamma_{q_{0}}$,

$$
\begin{aligned}
& \left|T_{\vec{\lambda}}(F)\left(\rho_{1} y_{1}, \rho_{2} y_{2}\right)-T_{\vec{q}}^{(p)}(F)\left(\rho_{1} y_{1}, \rho_{2} y_{2}\right)\right|^{p^{\prime}} \\
& \leq \mid \int_{C_{a, b}^{\prime}[0, T]} \exp \left\{\sum_{j=1}^{2} i \rho_{j}\left(A_{j}^{1 / 2} w, y_{j}\right)^{\sim}\right\} \\
& \quad \times\left.[\psi(\vec{\lambda} ; \vec{A} ; w)-\psi(-i \vec{q} ; \vec{A} ; w)] d f(w)\right|^{p^{\prime}} \\
& \leq\left(\int_{C_{a, b}^{\prime}[0, T]}[|\psi(\vec{\lambda} ; \vec{A} ; w)|+|\psi(-i \vec{q} ; \vec{A} ; w)|] d|f|(w)\right)^{p^{\prime}} \\
& \leq\left(2 \int_{C_{a, b}^{\prime}[0, T]} k\left(q_{0} ; \vec{A} ; w\right) d|f|(w)\right)^{p^{\prime}}<+\infty .
\end{aligned}
$$

Hence, by the dominated convergence theorem, we see that for each $p \in(1,2]$ and each $\rho_{1}>0$ and $\rho_{2}>0$,

$$
\begin{array}{r}
\lim _{\vec{\lambda} \rightarrow-i \vec{q}} \int_{C_{a, b}^{2}[0, T]} \mid T_{\vec{\lambda}}(F)\left(\rho_{1} y_{1}, \rho_{2} y_{2}\right) \\
-\left.T_{\vec{q}}^{(p)}(F)\left(\rho_{1} y_{1}, \rho_{2} y_{2}\right)\right|^{p^{\prime}} d(\mu \times \mu)\left(y_{1}, y_{2}\right) \\
=\lim _{\vec{\lambda} \rightarrow-i \vec{q}} \int_{C_{a, b}^{2}[0, T]} \mid \int_{C_{a, b}^{\prime}[0, T]} \exp \left\{\sum_{j=1}^{2} i\left(A_{j}^{1 / 2} w, \rho_{j} y_{j}\right)^{\sim}\right\} \\
\quad \times \psi(\vec{\lambda} ; \vec{A} ; w) d f(w) \\
-\int_{C_{a, b}^{\prime}[0, T]} \exp \left\{\sum_{j=1}^{2} i\left(A_{j}^{1 / 2} w, \rho_{j} y_{j}\right)^{\sim}\right\} \\
\times\left.\psi(-i \vec{q} ; \vec{A} ; w) d f(w)\right|^{p^{\prime}} \\
\times d(\mu \times \mu)\left(y_{1}, y_{2}\right)
\end{array}
$$

$$
\begin{aligned}
& =\int_{C_{a, b}^{2}[0, T]} \mid \int_{C_{a, b}^{\prime}[0, T]} \exp \left\{\sum_{j=1}^{2} i\left(A_{j}^{1 / 2} w, \rho_{j} y_{j}\right)^{\sim}\right\} \\
& \quad \times \lim _{\vec{\lambda} \rightarrow-i \vec{q}}[\psi(\vec{\lambda} ; \vec{A} ; w) \\
& -\psi(-i \vec{q} ; \vec{A} ; w)]\left.d f(w)\right|^{p^{\prime}} \\
& =0 \quad \times d(\mu \times \mu)\left(y_{1}, y_{2}\right)
\end{aligned}
$$

which concludes the proof of Theorem 10.

Remark 11. (1) In view of Theorems 9 and 10, we see that for each $p \in[1,2]$, the $L_{p}$ analytic GFFT of $F, T_{\vec{q}}^{(p)}(F)$ is given by the right hand side of (40) for $q_{0}, q_{1}, q_{2}$, and $F$ as in Theorem 9, and for s-a.e. $\left(y_{1}, y_{2}\right) \in C_{a, b}^{2}[0, T]$,

$$
T_{\vec{q}}^{(p)}(F)\left(y_{1}, y_{2}\right)=E_{\vec{x}}^{\operatorname{anf}_{\vec{q}}}\left[F\left(y_{1}+x_{1}, y_{2}+x_{2}\right)\right], \quad p \in[1,2] .
$$

In particular, using this fact and (29), we have that for all $p \in$ $[1,2]$,

$$
T_{\vec{q}}^{(p)}(F)(0,0)=E_{\vec{x}}^{\operatorname{anf}_{\vec{q}}}\left[F\left(x_{1}, x_{2}\right)\right] .
$$

(2) For nonzero real numbers $q_{1}$ and $q_{2}$ with $\left|q_{j}\right|>q_{0}$, $j \in\{1,2\}$, define a set function $f_{\vec{q}}^{\vec{A}}: \mathscr{B}\left(C_{a, b}^{\prime}[0, T]\right) \rightarrow \mathbb{C}$ by

$$
f_{\vec{q}}^{\vec{A}}(B)=\int_{B} \psi(-i \vec{q} ; \vec{A} ; w) d f(w), \quad B \in \mathscr{B}\left(C_{a, b}^{\prime}[0, T]\right),
$$

where $f$ and $F$ are related by $(30)$ and $\mathscr{B}\left(C_{a, b}^{\prime}[0, T]\right)$ is the Borel $\sigma$-algebra of $C_{a, b}^{\prime}[0, T]$. Then it is obvious that $f_{\vec{q}}^{\vec{A}}$ belongs to $\mathscr{M}\left(C_{a, b}^{\prime}[0, T]\right)$ and for all $p \in[1,2], T_{\vec{q}}^{(p)}(F)$ can be expressed as

$$
T_{\vec{q}}^{(p)}(F)\left(y_{1}, y_{2}\right)=\int_{C_{a, b}^{\prime}[0, T]} \exp \left\{\sum_{j=1}^{2} i\left(A_{j}^{1 / 2} w, y_{j}\right)^{\sim}\right\} d f_{\vec{q}}^{\vec{A}}(w)
$$

for s-a.e. $\left(y_{1}, y_{2}\right) \in C_{a, b}^{2}[0, T]$. Hence, $T_{\vec{q}}^{(p)}(F)$ belongs to $\mathscr{F}_{A_{1}, A_{2}}^{a, b}$ for all $p \in[1,2]$.

\section{Relationships between the GFFT and the Function Space Integral of Functionals in $\mathscr{F}_{A_{1}, A_{2}}^{a, b}$}

In this section we establish a relationship between the GFFT and the function space integral of functionals in the Fresnel type class $\mathscr{F}_{A_{1}, A_{2}}^{a, b}$. 
Throughout this section, for convenience, we use the following notation: for given $\lambda \in \widetilde{\mathbb{C}}_{+}$and $n=1,2, \ldots$, let

$$
\begin{aligned}
& G_{n}(\lambda, x) \\
& =\exp \left\{\left[\frac{1-\lambda}{2}\right] \sum_{k=1}^{n}\left[\left(e_{k}, x\right)^{\sim}\right]^{2}\right. \\
& \left.+\left(\lambda^{1 / 2}-1\right) \sum_{k=1}^{n}\left(e_{k}, a\right)_{C_{a, b}^{\prime}}\left(e_{k}, x\right)^{\sim}\right\},
\end{aligned}
$$

where $\left\{e_{n}\right\}_{n=1}^{\infty}$ is a complete orthonormal set in $\left(C_{a, b}^{\prime}[0, T]\right.$, $\left.\|\cdot\|_{b}\right)$.

To obtain our main results, Theorems 14 and 17 below, we state a fundamental integration formula for the function space $C_{a, b}[0, T]$.

Let $\left\{e_{1}, \ldots, e_{n}\right\}$ be an orthonormal set in $\left(C_{a, b}^{\prime}[0, T],\|\cdot\|_{b}\right)$, let $k: \mathbb{R}^{n} \rightarrow \mathbb{C}$ be a Lebesgue measurable function, and let $K: C_{a, b}[0, T] \rightarrow \mathbb{C}$ be given by

$$
K(x)=k\left(\left(e_{1}, x\right)^{\sim}, \ldots,\left(e_{n}, x\right)^{\sim}\right) .
$$

Then

$$
\begin{aligned}
& E[K]= \int_{C_{a, b}[0, T]} k\left(\left(e_{1}, x\right)^{\sim}, \ldots,\left(e_{n}, x\right)^{\sim}\right) d \mu(x) \\
&=(2 \pi)^{-n / 2} \int_{\mathbb{R}^{n}} k\left(u_{1}, \ldots, u_{n}\right) \\
& \times \exp \left\{-\sum_{j=1}^{n} \frac{\left[u_{j}-\left(e_{j}, a\right)_{b}\right]^{2}}{2}\right\} d u_{1} \ldots d u_{n}
\end{aligned}
$$

in the sense that if either side of (54) exists, both sides exist and equality holds.

We also need the following lemma to obtain our main theorem in this section.

Lemma 12. Let $\left\{e_{1}, \ldots, e_{n}\right\}$ be an orthonormal subset of $\left(C_{a, b}^{\prime}[0, T],\|\cdot\|_{b}\right)$ and let $w$ be an element of $C_{a, b}^{\prime}[0, T]$. Then for each $\lambda \in \mathbb{C}_{+}$, the function space integral

$$
E_{x}\left[G_{n}(\lambda, x) \exp \left\{i(w, x)^{\sim}\right\}\right]
$$

exists and is given by the formula

$$
\begin{aligned}
& E_{x}\left[G_{n}(\lambda, x) \exp \{\right.\left.\left.i(w, x)^{\sim}\right\}\right] \\
&=\lambda^{-n / 2} \exp \left\{\left[\frac{\lambda-1}{2 \lambda}\right] \sum_{k=1}^{n}\left(e_{k}, w\right)_{b}^{2}-\frac{1}{2}\|w\|_{b}^{2}\right. \\
&+i \lambda^{-1 / 2} \sum_{k=1}^{n}\left(e_{k}, a\right)_{b}\left(e_{k}, w\right)_{b} \\
&\left.+i\left(e_{n+1}, a\right)_{b}\left[\|w\|_{b}^{2}-\sum_{k=1}^{n}\left(e_{k}, w\right)_{b}^{2}\right]^{1 / 2}\right\},
\end{aligned}
$$

where $G_{n}$ is given by (52) above and

$$
e_{n+1}=\left[\|w\|_{b}^{2}-\sum_{j=1}^{n}\left(e_{j}, w\right)_{b}^{2}\right]^{-1 / 2}\left\{w-\sum_{j=1}^{n}\left(e_{j}, w\right)_{b} e_{j}\right\} .
$$

Proof(Outline). Using the Gram-Schmidt process, for any $w \in C_{a, b}^{\prime}[0, T]$, we can write $w=\sum_{k=1}^{n+1} c_{k} e_{k}$, where $\left\{e_{1}, \ldots, e_{n}, e_{n+1}\right\}$ is an orthonormal set in $\left(C_{a, b}^{\prime}[0, T],\|\cdot\|_{b}\right)$ and

$$
c_{k}= \begin{cases}\left(e_{k}, w\right)_{b}, & k=1, \ldots, n \\ {\left[\|w\|_{b}^{2}-\sum_{j=1}^{n}\left(e_{j}, w\right)_{b}^{2}\right]^{1 / 2},} & k=n+1 .\end{cases}
$$

Then using (52), (54), the Fubini theorem, and (14), it follows that (56) holds for all $\lambda \in \mathbb{C}_{+}$.

The following remark will be very useful in the proof of our main theorem in this section.

Remark 13. Let $q_{0}$ be a positive real number and let $\Gamma_{q_{0}}$ be given by (35). For real numbers $q_{1}$ and $q_{2}$ with $\left|q_{j}\right|>q_{0}, j \in$ $\{1,2\}$, let $\left\{\vec{\lambda}_{n}\right\}_{n=1}^{\infty}=\left\{\left(\lambda_{1, n}, \lambda_{2, n}\right)\right\}_{n=1}^{\infty}$ be a sequence in $\mathbb{C}_{+}^{2}$ such that

$$
\vec{\lambda}_{n}=\left(\lambda_{1, n}, \lambda_{2, n}\right) \longrightarrow-i \vec{q}=\left(-i q_{1},-i q_{2}\right) .
$$

Let $\lambda_{j, n}=\alpha_{j, n}+i \beta_{j, n}$ for $j \in\{1,2\}$ and $n \in \mathbb{N}$. Then for $j \in\{1,2\}, \operatorname{Re}\left(\lambda_{j, n}\right)=\alpha_{j, n}>0$ and

$$
\lambda_{j, n}^{-1}=\left(\alpha_{j, n}+i \beta_{j, n}\right)^{-1}=\frac{\alpha_{j, n}-i \beta_{j, n}}{\alpha_{j, n}^{2}+\beta_{j, n}^{2}}
$$

for each $n \in \mathbb{N}$. Since $\left|\operatorname{Im}\left(\left(-i q_{j}\right)^{-1 / 2}\right)\right|=1 / \sqrt{2\left|q_{j}\right|}<1 / \sqrt{2 q_{0}}$ for $j \in\{1,2\}$, there exists a sufficiently large $L \in \mathbb{N}$ such that for any $n \geq L, \lambda_{1, n}$ and $\lambda_{2, n}$ are in $\operatorname{Int}\left(\Gamma_{q_{0}}\right)$ and

$$
\begin{aligned}
\delta\left(q_{1}, q_{2}\right) \equiv \sup ( & \left\{\left|\operatorname{Im}\left(\lambda_{1, n}^{-1 / 2}\right)\right|: n \geq L\right\} \\
& \cup\left\{\left|\operatorname{Im}\left(\lambda_{2, n}^{-1 / 2}\right)\right|: n \geq L\right\} \\
& \cup\left\{\left|\operatorname{Im}\left(\left(-i q_{1}\right)^{-1 / 2}\right)\right|,\right. \\
<\frac{1}{\sqrt{2 q_{0}}} . & \left.\left.\left|\operatorname{Im}\left(\left(-i q_{2}\right)^{-1 / 2}\right)\right|\right\}\right)
\end{aligned}
$$

Thus, there exists a positive real number $\varepsilon>1$ such that $\delta\left(q_{1}, q_{2}\right)<1 /\left(\varepsilon \sqrt{2 q_{0}}\right)$.

Let $\left\{e_{n}\right\}_{n=1}^{\infty}$ be a complete orthonormal set in $\left(C_{a, b}^{\prime}[0, T]\right.$, $\left.\|\cdot\|_{b}\right)$. Using Parseval's identity, it follows that

$$
\left(g_{1}, g_{2}\right)_{b}=\sum_{n=1}^{\infty}\left(e_{n}, g_{1}\right)_{b}\left(e_{n}, g_{2}\right)_{b}
$$


for all $g_{1}, g_{2} \in C_{a, b}^{\prime}[0, T]$. In addition for each $g \in C_{a, b}^{\prime}[0, T]$,

$$
\|g\|_{b}^{2}-\sum_{k=1}^{n}\left(e_{k}, g\right)_{b}^{2}=\sum_{k=n+1}^{\infty}\left(e_{k}, g\right)_{b}^{2} \geq 0
$$

for every $n \in \mathbb{N}$.

Since

$$
(g, a)_{b}=\sum_{n=1}^{\infty}\left(e_{n}, g\right)_{b}\left(e_{n}, a\right)_{b}
$$

and for $\varepsilon>1$

$$
\begin{aligned}
-\varepsilon\|g\|_{b}\|a\|_{b} & <-\|g\|_{b}\|a\|_{b} \leq(g, a)_{b} \\
& \leq\|g\|_{b}\|a\|_{b}<\varepsilon\|g\|_{b}\|a\|_{b},
\end{aligned}
$$

there exists a sufficiently large $K_{j} \in \mathbb{N}$ such that for any $n \geq K_{j}$

$$
\left|\sum_{k=1}^{n}\left(e_{k}, A_{j}^{1 / 2} w\right)_{b}\left(e_{k}, a\right)_{b}\right|<\varepsilon\left\|A_{j}^{1 / 2} w\right\|_{b}\|a\|_{b}
$$

for $j \in\{1,2\}$.

Using these and a long and tedious calculation we can show that for every $n \geq \max \left\{L, K_{1}, K_{2}\right\}$,

$$
\begin{aligned}
& \mid \exp \left\{\sum_{j=1}^{2}(\right. {\left[\frac{\lambda_{j, n}-1}{2 \lambda_{j, n}}\right] \sum_{k=1}^{n}\left(e_{k}, A_{j}^{1 / 2} w\right)_{b}^{2}-\frac{1}{2}\left\|A_{j}^{1 / 2} w\right\|_{b}^{2} } \\
&+i \lambda_{j, n}^{-1 / 2} \sum_{k=1}^{n}\left(e_{k}, A_{j}^{1 / 2} w\right)_{b}\left(e_{k}, a\right)_{b}+i\left(e_{n+1}, a\right)_{b} \\
&\left.\left.\times\left[\left\|A_{j}^{1 / 2} w\right\|_{b}^{2}-\sum_{k=1}^{n}\left(e_{k}, A_{j}^{1 / 2} w\right)_{b}^{2}\right]^{1 / 2}\right)\right\} \mid \\
&<k\left(q_{0} ; \vec{A} ; w\right),
\end{aligned}
$$

where $k\left(q_{0} ; \vec{A} ; w\right)$ is given by (36).

In our next theorem, for $F \in \underset{A_{1}, A_{2}}{a, b}$, we express the GFFT of $F$ as the limit of a sequence of function space integrals on $C_{a, b}^{2}[0, T]$.

Theorem 14. Let $q_{0}$ and $F$ be as in Theorem 10. Let $\left\{e_{n}\right\}_{n=1}^{\infty}$ be a complete orthonormal set in $\left(C_{a, b}^{\prime}[0, T],\|\cdot\|_{b}\right)$ and let $\left\{\left(\lambda_{1, n}, \lambda_{2, n}\right)\right\}_{n=1}^{\infty}$ be a sequence in $\mathbb{C}_{+}^{2}$ such that $\lambda_{j, n} \rightarrow-i q_{j}$ where $q_{j}$ is a real number with $\left|q_{j}\right|>q_{0}, j \in\{1,2\}$. Then for $p \in[1,2]$ and for s-a.e. $\left(y_{1}, y_{2}\right) \in C_{a, b}^{2}[0, T]$,

$$
\begin{aligned}
T_{\vec{q}}^{(p)}(F) & \left(y_{1}, y_{2}\right) \\
= & \lim _{n \rightarrow \infty} \lambda_{1, n}^{n / 2} \lambda_{2, n}^{n / 2} \\
& \times E_{\vec{x}}\left[G_{n}\left(\lambda_{1, n}, x_{1}\right) G_{n}\left(\lambda_{2, n}, x_{2}\right) F\left(y_{1}+x_{1}, y_{2}+x_{2}\right)\right],
\end{aligned}
$$

where $G_{n}$ is given by (52).
Proof. By Theorems 9 and 10, we know that for each $p \in[1,2]$, the $L_{p}$ analytic GFFT of $F, T_{\vec{q}}^{(p)}(F)$ exists and is given by the right hand side of (40). Thus, it suffices to show that

$$
\begin{aligned}
T_{\vec{q}}^{(1)}(F)\left(y_{1}, y_{2}\right)= & E_{\vec{x}}^{\operatorname{anf}_{\vec{q}}}\left[F\left(y_{1}+x_{1}, y_{2}+x_{2}\right)\right] \\
= & \lim _{n \rightarrow \infty} \lambda_{1, n}^{n / 2} \lambda_{2, n}^{n / 2} \\
& \times E_{\vec{x}}\left[G_{n}\left(\lambda_{1, n}, x_{1}\right) G_{n}\left(\lambda_{2, n}, x_{2}\right)\right. \\
& \left.\times F\left(y_{1}+x_{1}, y_{2}+x_{2}\right)\right] .
\end{aligned}
$$

Using (30), the Fubini theorem and (56) with $\lambda$ and $w$ replaced with $\lambda_{j, n}$ and $A_{j}^{1 / 2} w, j \in\{1,2\}$, respectively, we see that

$$
\begin{gathered}
\lambda_{1, n}^{n / 2} \lambda_{2, n}^{n / 2} E_{\vec{x}}\left[G_{n}\left(\lambda_{1, n}, x_{1}\right) G_{n}\left(\lambda_{2, n}, x_{2}\right) F\left(y_{1}+x_{1}, y_{2}+x_{2}\right)\right] \\
=\lambda_{1, n}^{n / 2} \lambda_{2, n}^{n / 2} \int_{C_{a, b}^{\prime}[0, T]} \exp \left\{\sum_{j=1}^{2} i\left(A_{j}^{1 / 2} w, y_{j}\right)^{\sim}\right\} \\
\times\left(\prod _ { j = 1 } ^ { 2 } E _ { x _ { j } } \left[G_{n}\left(\lambda_{j, n}^{-1 / 2}, x_{j}\right)\right.\right. \\
\left.\left.\quad \times \exp \left\{i\left(A_{j}^{1 / 2} w, x_{j}\right)^{\sim}\right\}\right]\right) d f(w) \\
=\int_{C_{a, b}^{\prime}[0, T]} \exp \left\{\sum _ { j = 1 } ^ { 2 } \left(i\left(A_{j}^{1 / 2} w, y_{j}\right)^{\sim}\right.\right. \\
+\left[\frac{\lambda_{j, n}-1}{2 \lambda_{j, n}}\right] \sum_{k=1}^{n}\left(e_{k}, A_{j}^{1 / 2} w\right)_{b}^{2} \\
-\frac{1}{2}\left\|A_{j}^{1 / 2} w\right\|_{b}^{2} \\
+i \lambda_{j, n}^{-1 / 2} \sum_{k=1}^{n}\left(e_{k}, a\right)_{b}\left(e_{k}, A_{j}^{1 / 2} w\right)_{b} \\
+i\left(e_{n+1}, a\right)_{b}\left[\left\|A_{j}^{1 / 2} w\right\|_{b}^{2}\right. \\
\left.\left.\left.-\sum_{k=1}^{n}\left(e_{k}, A_{j}^{1 / 2} w\right)_{b}^{2}\right]^{1 / 2}\right)\right\} d f(w) .
\end{gathered}
$$

But, by Remark 13 we see that the last expression of (70) is dominated by (39) on the region $\Gamma_{q_{0}}$ given by (35) for all but a finite number of values of $n$. Next using the dominated convergence theorem, Parseval's relation and (40), we obtain the desired result.

Corollary 15. Let $q_{0}, F,\left\{e_{n}\right\}_{n=1}^{\infty},\left\{\left(\lambda_{1, n}, \lambda_{2, n}\right)\right\}_{n=1}^{\infty}$ and $\left(q_{1}, q_{2}\right)$ be as in Theorem 14. Then

$$
\begin{aligned}
E_{\vec{x}}^{a n f} f_{\vec{q}} & {\left[F\left(x_{1}, x_{2}\right)\right] } \\
= & \lim _{n \rightarrow \infty} \lambda_{1, n}^{n / 2} \lambda_{2, n}^{n / 2} \\
& \times E_{\vec{x}}\left[G_{n}\left(\lambda_{1, n}, x_{1}\right) G_{n}\left(\lambda_{2, n}, x_{2}\right) F\left(x_{1}, x_{2}\right)\right],
\end{aligned}
$$

where $G_{n}$ is given by (52). 
Corollary 16. Let $q_{0}, F$ and $\left\{e_{n}\right\}_{n=1}^{\infty}$ be as in Theorem 14 and let $\Gamma_{q_{0}}$ be given by (35). Let $\vec{\lambda}=\left(\lambda_{1}, \lambda_{2}\right) \in \operatorname{Int}\left(\Gamma_{q_{0}}\right)$ and $\left\{\left(\lambda_{1, n}, \lambda_{2, n}\right)\right\}_{n=1}^{\infty}$ be a sequence in $\mathbb{C}_{+}^{2}$ such that $\lambda_{j, n} \rightarrow \lambda_{j}$, $j \in\{1,2\}$. Then

$$
\begin{aligned}
E_{\vec{x}}^{a n_{\vec{\lambda}}}\left[F\left(x_{1}, x_{2}\right)\right]= & \lim _{n \rightarrow \infty} \lambda_{1, n}^{n / 2} \lambda_{2, n}^{n / 2} \\
& \times E_{\vec{x}}\left[G_{n}\left(\lambda_{1, n}, x_{1}\right) G_{n}\left(\lambda_{2, n}, x_{2}\right) F\left(x_{1}, x_{2}\right)\right],
\end{aligned}
$$

where $G_{n}$ is given by (52).

Our another result, namely, a change of scale formula for function space integrals now follows from Corollary 16 above.

Theorem 17. Let $F \in \mathscr{F}_{A_{1}, A_{2}}^{a, b}$ and let $\left\{e_{n}\right\}_{n=1}^{\infty}$ be a complete orthonormal set in $\left(C_{a, b}^{\prime}[0, T],\|\cdot\|_{b}\right)$. Then for any $\rho_{1}>0$ and $\rho_{2}>0$,

$$
\begin{aligned}
E_{\vec{x}}[ & \left.F\left(\rho_{1} x_{1}, \rho_{2} x_{2}\right)\right] \\
= & \lim _{n \rightarrow \infty} \rho_{1}^{-n} \rho_{2}^{-n} \\
& \times E_{\vec{x}}\left[G_{n}\left(\rho_{1}^{-2}, x_{1}\right) G_{n}\left(\rho_{2}^{-2}, x_{2}\right) F\left(x_{1}, x_{2}\right)\right],
\end{aligned}
$$

where $G_{n}$ is given by (52).

Proof. Simply choose $\lambda_{j}=\rho_{j}^{-2}$ for $j \in\{1,2\}$ and $\lambda_{j, n}=\rho_{j}^{-2}$ for $j \in\{1,2\}$ and $n \in \mathbb{N}$ in (72).

Remark 18. Of course, if we choose $a(t) \equiv 0, b(t)=t$, $A_{1}=I$ (identity operator), and $A_{2}=0$ (zero operator), then the function space $C_{a, b}[0, T]$ reduces to the classical Wiener space $C_{0}[0, T]$ and the generalized Fresnel type class $\mathscr{F}_{A_{1}, A_{2}}^{a, b}$ reduces to the Fresnel class $\mathscr{F}\left(C_{0}[0, T]\right)$. It is known that $\mathscr{F}\left(C_{0}[0, T]\right)$ forms a Banach algebra over the complex field. In this case, we have the relationships between the analytic Feynman integral and the Wiener integral on classical Wiener space as discussed in $[14,15]$.

In recent paper [19], Yoo et al. have studied a change of scale formula for function space integral of the functionals in the Banach algebra $\delta\left(L_{a, b}^{2}[0, T]\right)$; the Banach algebra $\mathcal{S}\left(L_{a, b}^{2}[0, T]\right)$ is introduced in [12].

\section{Functionals in $\mathscr{F}_{A_{1}, A_{2}}^{a, b}$}

In this section, we prove a theorem ensuring that various functionals are in $\mathscr{F}_{A_{1}, A_{2}}^{a, b}$.

Theorem 19. Let $A_{1}$ and $A_{2}$ be bounded, nonnegative, and self-adjoint operators on $C_{a, b}^{\prime}[0, T]$. Let $(Y, \mathcal{Y}, \gamma)$ be a $\sigma$-finite measure space and let $\varphi_{l}: Y \rightarrow C_{a, b}^{\prime}[0, T]$ be $\mathscr{Y}-\mathscr{B}\left(C_{a, b}^{\prime}[0, T]\right)$ measurable for $l \in\{1, \ldots, d\}$. Let $\theta: Y \times \mathbb{R}^{d} \rightarrow \mathbb{C}$ be given by $\theta(\eta ; \cdot)=\widehat{v}_{\eta}(\cdot)$, where $\nu_{\eta} \in \mathscr{M}\left(\mathbb{R}^{d}\right)$ for every $\eta \in Y$ and where the family $\left\{v_{\eta}: \eta \in Y\right\}$ satisfies

(i) $\nu_{\eta}(E)$ is a $\mathscr{Y}$-measurable function of $\eta$ for every $E \in$ $\mathscr{B}\left(\mathbb{R}^{d}\right)$;

(ii) $\left\|\nu_{\eta}\right\| \in L^{1}(Y, \mathscr{Y}, \gamma)$.
Under these hypothesis, the functional $F: C_{a, b}^{2}[0, T] \rightarrow \mathbb{C}$ given by

$$
\begin{gathered}
F\left(x_{1}, x_{2}\right)=\int_{Y} \theta\left(\eta ; \sum_{j=1}^{2}\left(A_{j}^{1 / 2} \varphi_{1}(\eta), x_{j}\right)^{\sim},\right. \\
\left.\ldots, \sum_{j=1}^{2}\left(A_{j}^{1 / 2} \varphi_{d}(\eta), x_{j}\right)^{\sim}\right) d \gamma(\eta)
\end{gathered}
$$

belongs to $\mathscr{F}_{A_{1}, A_{2}}^{a, b}$ and satisfies the inequality

$$
\|F\| \leq \int_{Y}\left\|v_{\eta}\right\| d \gamma(\eta)
$$

Proof. Using the techniques similar to those used in [20], we can show that $\left\|\nu_{\eta}\right\|$ is measurable as a function of $\eta$, that $\theta$ is $\mathscr{Y}$-measurable, and that the integrand in (74) is a measurable function of $\eta$ for every $\left(x_{1}, x_{2}\right) \in C_{a, b}^{2}[0, T]$.

We define a measure $\tau$ on $\mathscr{Y} \times \mathscr{B}\left(\mathbb{R}^{d}\right)$ by

$$
\tau(E)=\int_{Y} \nu_{\eta}\left(E^{(\eta)}\right) d \gamma(\eta), \quad \text { for } E \in \mathscr{Y} \times \mathscr{B}\left(\mathbb{R}^{d}\right) .
$$

Then by the first assertion of Theorem 3.1 in [17], $\tau$ satisfies $\|\tau\| \leq \int_{Y}\left\|\nu_{\eta}\right\| d \gamma(\eta)$. Now let $\Phi: Y \times \mathbb{R}^{d} \rightarrow C_{a, b}^{\prime}[0, T]$ be defined by $\Phi\left(\eta ; v_{1}, \ldots, v_{d}\right)=\sum_{l=1}^{d} v_{l} \varphi_{l}(\eta)$. Then $\Phi$ is $\mathcal{Y} \times$ $\mathscr{B}\left(\mathbb{R}^{d}\right)-\mathscr{B}\left(C_{a, b}^{\prime}[0, T]\right)$-measurable on the hypothesis for $\varphi_{l}$, $l \in\{1, \ldots, d\}$. Let $\sigma=\tau \circ \Phi^{-1}$. Then clearly $\sigma \in \mathscr{M}\left(C_{a, b}^{\prime}[0, T]\right)$ and satisfies $\|\sigma\| \leq\|\tau\|$.

From the change of variables theorem and the second assertion of Theorem 3.1 in [17], it follows that for a.e. $\left(x_{1}, x_{2}\right) \in C_{a, b}^{2}[0, T]$ and for every $\rho_{1}>0$ and $\rho_{2}>0$,

$$
\begin{aligned}
& F\left(\rho_{1} x_{1}, \rho_{2} x_{2}\right) \\
& =\int_{Y} \widehat{v}_{\eta}\left(\sum_{j=1}^{2}\left(A_{j}^{1 / 2} \varphi_{1}(\eta), \rho_{j} x_{j}\right)^{\sim},\right. \\
& \left.\ldots, \sum_{j=1}^{2}\left(A_{j}^{1 / 2} \varphi_{d}(\eta), \rho_{j} x_{j}\right)^{\sim}\right) d \gamma(\eta) \\
& =\int_{Y}\left[\int _ { \mathbb { R } ^ { d } } \operatorname { e x p } \left\{i \sum _ { l = 1 } ^ { d } v _ { l } \left[\sum _ { j = 1 } ^ { 2 } \left(A_{j}^{1 / 2} \varphi_{l}(\eta),\right.\right.\right.\right. \\
& \left.\quad \times\left(v_{1}, \ldots, v_{d}\right)\right] d \gamma(\eta) \\
& =\int_{Y \times \mathbb{R}^{d}} \exp \left\{i \sum_{l=1}^{d} v_{l}\left[\sum_{j=1}^{2}\left(A_{j}^{1 / 2} \varphi_{l}(\eta), \rho_{j} x_{j}\right)^{\sim}\right]\right\} d \tau \\
& \quad \times\left(\eta ; v_{1}, \ldots, v_{d}\right)
\end{aligned}
$$




$$
\begin{aligned}
& =\int_{Y \times \mathbb{R}^{d}} \exp \left\{\sum_{j=1}^{2} i\left(A_{j}^{1 / 2} \Phi\left(\eta ; v_{1}, \ldots, v_{d}\right), \rho_{j} x_{j}\right)^{\sim}\right\} d \tau \\
& \quad \times\left(\eta ; v_{1}, \ldots, v_{d}\right) \\
& =\int_{C_{a, b}^{\prime}[0, T]} \exp \left\{\sum_{j=1}^{2} i\left(A_{j}^{1 / 2} w, \rho_{j} x_{j}\right)^{\sim}\right\} d \tau \circ \Phi^{-1}(w) \\
& =\int_{C_{a, b}^{\prime}[0, T]} \exp \left\{\sum_{j=1}^{2} i\left(A_{j}^{1 / 2} w, \rho_{j} x_{j}\right)^{\sim}\right\} d \sigma(w) .
\end{aligned}
$$

Thus, the functional $F$ given by (74) belongs to $\mathscr{F}_{A_{1}, A_{2}}^{a, b}$ and satisfies the inequality

$$
\|F\|=\|\sigma\| \leq\|\tau\| \leq \int_{Y}\left\|v_{\eta}\right\| d \gamma(\eta) .
$$

As mentioned in (2) of Remark 6, $\mathscr{F}_{A_{1}, A_{2}}^{a, b}$ is a Banach algebra if $\operatorname{Ran}\left(A_{1}+A_{2}\right)$ is dense in $C_{a, b}^{\prime}[0, T]$. In this case, many analytic functionals of $F$ can be formed. The following corollary is relevant to Feynman integration theories and quantum mechanics where exponential functions play an important role.

Corollary 20. Let $A_{1}$ and $A_{2}$ be bounded, nonnegative and self-adjoint operators on $C_{a, b}^{\prime}[0, T]$ such that $\operatorname{Ran}\left(A_{1}+A_{2}\right)$ is dense in $C_{a, b}^{\prime}[0, T]$. Let $F$ be given by (74) with $\theta$ as in Theorem 19, and let $\beta: \mathbb{C} \rightarrow \mathbb{C}$ be an entire function. Then $(\beta \circ F)\left(x_{1}, x_{2}\right)$ is in $\mathscr{F}_{A_{1}, A_{2}}^{a, b}$. In particular, $\exp \left\{F\left(x_{1}, x_{2}\right)\right\} \in$ $\mathscr{F}_{A_{1}, A_{2}}^{a, b}$.

Corollary 21. Let $A_{1}$ and $A_{2}$ be bounded, nonnegative, and self-adjoint operators on $C_{a, b}^{\prime}[0, T]$, and let $\left\{g_{1}, \ldots, g_{d}\right\}$ be a finite subset of $C_{a, b}^{\prime}[0, T]$. Given $\beta=\widehat{v}$ where $v \in \mathscr{M}\left(\mathbb{R}^{d}\right)$, define $F: C_{a, b}^{2}[0, T] \rightarrow \mathbb{C}$ by

$$
F\left(x_{1}, x_{2}\right)=\beta\left(\sum_{j=1}^{2}\left(A_{j}^{1 / 2} g_{1}, x_{j}\right)^{\sim}, \ldots, \sum_{j=1}^{2}\left(A_{j}^{1 / 2} g_{d}, x_{j}\right)^{\sim}\right)
$$

Then $F$ is an element of $\mathscr{F}_{A_{1}, A_{2}}^{a, b}$.

Proof. Let $(Y, \mathcal{Y}, \gamma)$ be a probability space, and for $l \in$ $\{1, \ldots, d\}$, let $\varphi_{l}(\eta) \equiv g_{l}$. Take $\theta(\eta ; \cdot)=\beta(\cdot)=\widehat{\nu}(\cdot)$. Then for all $\rho_{1}>0$ and $\rho_{2}>0$ and for a.e. $\left(x_{1}, x_{2}\right) \in C_{a, b}^{2}[0, T]$,

$$
\begin{gathered}
\int_{Y} \theta\left(\eta ; \sum_{j=1}^{2}\left(A_{j}^{1 / 2} \varphi_{1}(\eta), \rho_{j} x_{j}\right)^{\sim},\right. \\
\left.\ldots, \sum_{j=1}^{2}\left(A_{j}^{1 / 2} \varphi_{d}(\eta), \rho_{j} x_{j}\right)^{\sim}\right) d \gamma(\eta)
\end{gathered}
$$

$$
\begin{aligned}
& =\int_{Y} \beta\left(\sum_{j=1}^{2}\left(A_{j}^{1 / 2} g_{1}, \rho_{j} x_{j}\right)^{\sim},\right. \\
& \left.\ldots, \sum_{j=1}^{2}\left(A_{j}^{1 / 2} g_{d}, \rho_{j} x_{j}\right)^{\sim}\right) d \gamma(\eta) \\
& =\beta\left(\sum_{j=1}^{2}\left(A_{j}^{1 / 2} g_{1}, \rho_{j} x_{j}\right)^{\sim}, \ldots, \sum_{j=1}^{2}\left(A_{j}^{1 / 2} g_{d}, \rho_{j} x_{j}\right)^{\sim}\right) \\
& =F\left(\rho_{1} x_{1}, \rho_{2} x_{2}\right) .
\end{aligned}
$$

Hence, $F \in \mathscr{F}_{A_{1}, A_{2}}^{a, b}$.

Remark 22. Let $d=1$ and let $(Y, \mathcal{Y}, \gamma)=([0, T], \mathscr{B}([0, T])$, $\left.m_{L}\right)$ in Theorem 19 where $m_{L}$ denotes the Lebesgue measure on $[0, T]$. Then Theorems 4.6, 4.7, and 4.9 in [18] follow from the results in this section by letting $A_{1}$ be the identity operator and letting $A_{2} \equiv 0$ on $C_{a, b}^{\prime}[0, T]$. The function $\theta$ studied in [18] (and mentioned in Remark 2 above) is interpreted as the potential energy in quantum mechanics.

\section{Acknowledgments}

The authors would like to express their gratitude to the referees for their valuable comments and suggestions which have improved the original paper. This research was supported by the Basic Science Research Program through the National Research Foundation of Korea (NRF) funded by the Ministry of Education (2011-0014552).

\section{References}

[1] G. Kallianpur and C. Bromley, "Generalized Feynman integrals using analytic continuation in several complex variables," in Stochastic Analysis and Applications, M. A. Pinsky, Ed., vol. 7, pp. 217-267, Marcel Dekker, New York, NY, USA, 1984.

[2] G. Kallianpur, D. Kannan, and R. L. Karandikar, "Analytic and sequential Feynman integrals on abstract Wiener and Hilbert spaces, and a Cameron-Martin formula," Annales de l'Institut Henri Poincaré, vol. 21, no. 4, pp. 323-361, 1985.

[3] G. W. Johnson and M. L. Lapidus, The Feynman Integral and Feynman's Operational Calculus, Clarendon Press, Oxford, UK, 2000.

[4] J. Yeh, "Singularity of Gaussian measures on function spaces induced by Brownian motion processes with non-stationary increments," Illinois Journal of Mathematics, vol. 15, pp. 37-46, 1971.

[5] J. Yeh, Stochastic Processes and the Wiener Integral, Marcel Dekker, New York, NY, USA, 1973.

[6] S. J. Chang, J. G. Choi, and D. Skoug, "Integration by parts formulas involving generalized Fourier-Feynman transforms on function space," Transactions of the American Mathematical Society, vol. 355, no. 7, pp. 2925-2948, 2003.

[7] S. J. Chang, J. G. Choi, and D. Skoug, "Parts formulas involving conditional generalized Feynman integrals and conditional generalized Fourier-Feynman transforms on function space," 
Integral Transforms and Special Functions, vol. 15, no. 6, pp. 491512, 2004.

[8] S. J. Chang, J. G. Choi, and D. Skoug, "Evaluation formulas for conditional function space integrals-I," Stochastic Analysis and Applications, vol. 25, no. 1, pp. 141-168, 2007.

[9] S. J. Chang, J. G. Choi, and D. Skoug, "Generalized FourierFeynman transforms, convolution products, and first variations on function space," The Rocky Mountain Journal of Mathematics, vol. 40, no. 3, pp. 761-788, 2010.

[10] S. J. Chang and D. M. Chung, "Conditional function space integrals with applications," The Rocky Mountain Journal of Mathematics, vol. 26, no. 1, pp. 37-62, 1996.

[11] S. J. Chang, H. S. Chung, and D. Skoug, "Integral transforms of functionals in $L_{2}\left(C_{a, b}[0, T]\right)$," The Journal of Fourier Analysis and Applications, vol. 15, no. 4, pp. 441-462, 2009.

[12] S. J. Chang and D. Skoug, "Generalized Fourier-Feynman transforms and a first variation on function space," Integral Transforms and Special Functions, vol. 14, pp. 375-393, 2003.

[13] J. G. Choi and S. J. Chang, "Generalized Fourier-Feynman transform and sequential transforms on function space," Journal of the Korean Mathematical Society, vol. 49, pp. 1065-1082, 2012.

[14] R. H. Cameron and D. A. Storvick, "Relationships between the Wiener integral and the analytic Feynman integral," Rendiconti del Circolo Matematico di Palermo, no. 17, supplement, pp. 117133, 1987.

[15] R. H. Cameron and D. A. Storvick, "Change of scale formulas for Wiener integral," Rendiconti del Circolo Matematico di Palermo, no. 17, pp. 105-115, 1987.

[16] G. W. Johnson, "The equivalence of two approaches to the Feynman integral," Journal of Mathematical Physics, vol. 23, pp. 2090-2096, 1982.

[17] G. W. Johnson and D. L. Skoug, "Notes on the Feynman integral-III: the Schroedinger equation," Pacific Journal of Mathematics, vol. 105, pp. 321-358, 1983.

[18] S. J. Chang, J. G. Choi, and S. D. Lee, "A Fresnel type class on function space," Journal of the Korean Society of Mathematical Education. Series B, vol. 16, no. 1, pp. 107-119, 2009.

[19] I. Yoo, B. J. Kim, and B. S. Kim, "A change of scale formula for a function space integral on $C_{a, b}[0, T]$," Proceedings of the American Mathematical Society, vol. 141, no. 8, pp. 2729-2739, 2013.

[20] K. S. Chang, G. W. Johnson, and D. L. Skoug, "Functions in the Fresnel class," Proceedings of the American Mathematical Society, vol. 100, no. 2, pp. 309-318, 1987. 


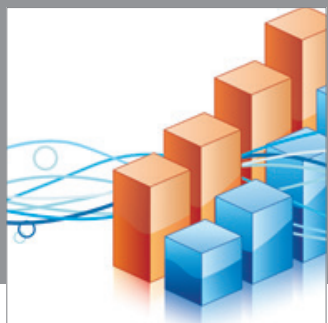

Advances in

Operations Research

mansans

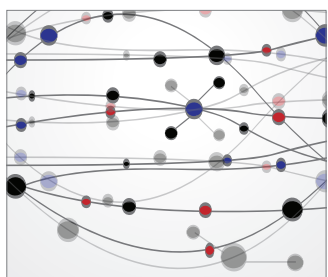

The Scientific World Journal
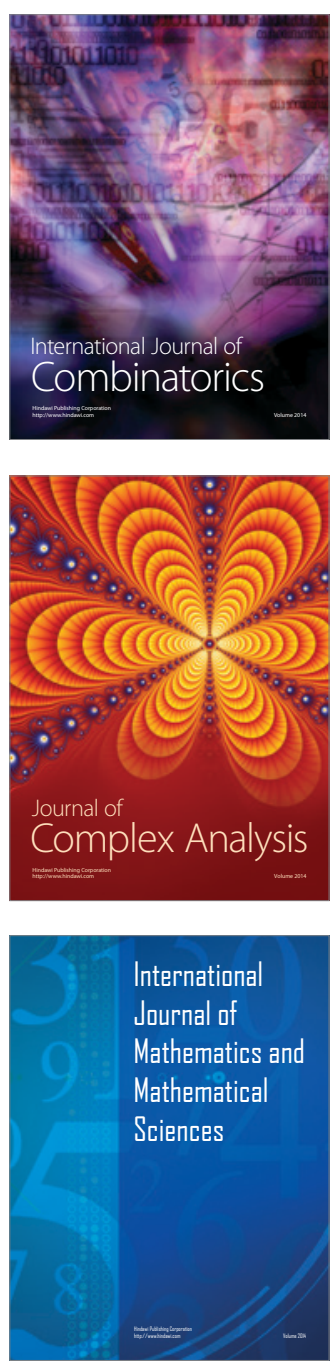


Submit your manuscripts at http://www.hindawi.com
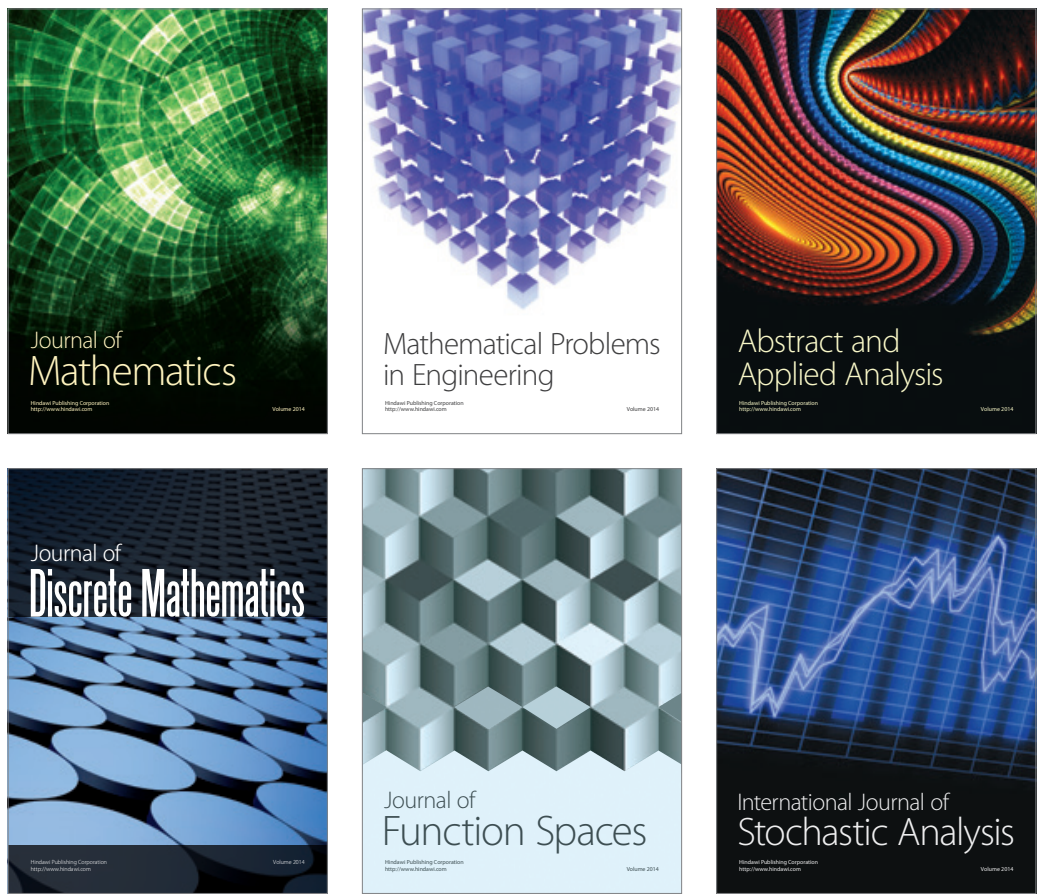

Journal of

Function Spaces

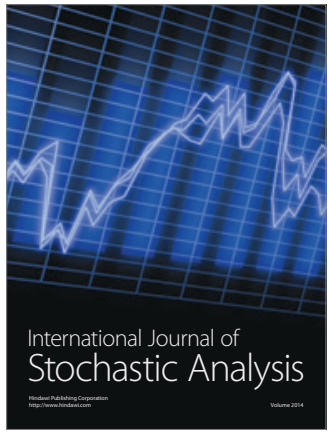

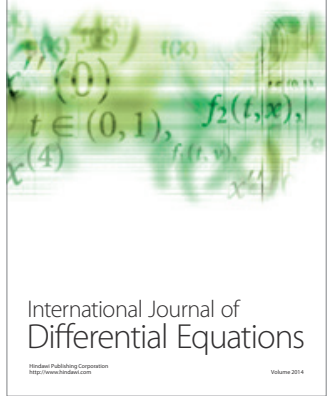
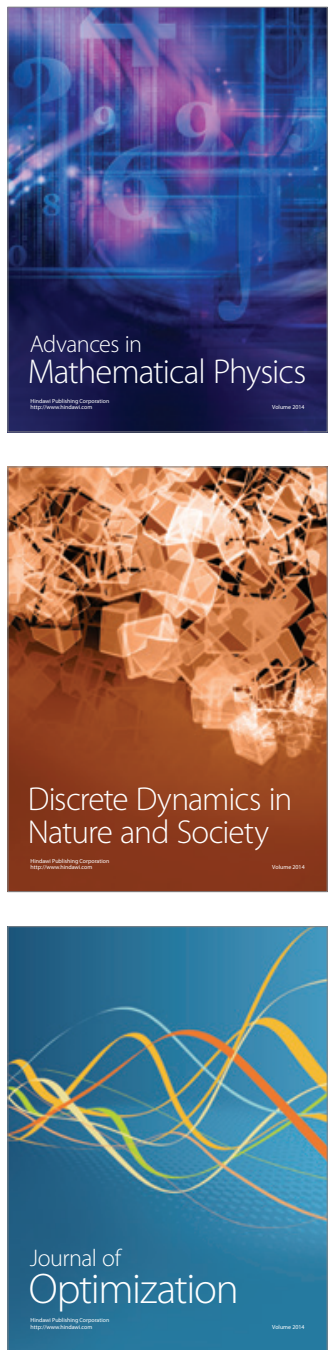